I kg. of pulverized $\mathrm{NaOH}$. When this has been well stirred in, add shredded asbestos gradually, continuing the stirring until the mixture no longer appears capable of wetting more asbestos. The whole mass is then heated in an air bath at I 50 to $180^{\circ}$ for $4 \mathrm{hrs}$. During the early part of the heating, additional quantities of asbestos are added from time to time until it presents the same appearance as before heating. After cooling, it is ground in a coffee mill until most of it passes a Io-mesh sieve. A tube filled with this absorbent takes up 8 to $\mathrm{I} \circ \mathrm{g}$. of carbon dioxide, corresponding to 160 to 240 carbon determinations, using the full factor weight on a steel containing 0.5 per cent carbon.

Research laboratory, Midvale Stegl Company Philatelifhia

\section{LABORATORY TABLE TOPS AGAIN}

\section{By HILTON IRA JONES}

Received August 17, 1916

Much has been written about various sorts of laboratory table tops-wood, alberene and tile advocates have all had their say. In the past twenty years I have worked and conducted classes on every sort of top-glass, tile, cement, slate, alberene stone and wood. When I came to Dakota Wesleyan I found a new one. Five years ago Dr. Sterling Temple, now of the University of Minnesota, installed the tables here. The tops are ordinary soft pine flooring on which is laid two sheets of tar paper, and this is covered with large sheets of asbestos slate, two to a table. It is oneeighth inch in thickness. The asbestos slate is screwed on, the holes being counter-sunk and filled above the screw head with asbestos cement. 'The tables every year have been treated with a gasoline solution of paraffin concentrated enough so that it would set to a jelly consistency when cold. This solution is brushed on hot. We have found this method of paraffining much better and quicker than the old ironing in process. This paraffin treatment has given the tops a polished appearance but is really unnecessary and adds nothing to their immediate serviceability or life.

This asbestos top is so much better than any other top I have ever seen that I am sure it would be extensively used if its advantages were more widely understood. It has low first cost, long life, and any one can put it on. I feel certain our table tops will be in good shape twenty years hence. They are fully as good now as when first installed. The asbestos top with the tar paper beneath it has elasticity, and I find there is even less glassware broken on it than on a wooden top. Alberene stone is especially objectionable for beginners for this reason. The asbestos slate top is entirely unaffected by acid or alkali even though boiling hot. Moreover, it is so poor a conductor of heat that even thick glass containing hot liquids may be set upon it without breaking. This does away with the use of suberite mats such as are required on stone, tile or glass tops. The asbestos slate top has everything to commend it, and after four years of use I have yet to discover a single fault.

DAKOTA WESLEYAAX UNIVERSITY

Mitcheli, South Dakota

\section{A SIMPLE MERCURY SEALED ETHER STILL}

By O. C. Smith and D. G. Morgan Received June 27,1916

The heater $H$ was constructed from a sheet of galvanized iron and an electric light socket fitted into the bottom. The corks which are inserted into the necks of the flasks at $D$ and $E$ should be close-grained and fit tightly so as to hold the mercury at $F$ and $G$. It is not difficult to make a mercury-tight fit if care is used in selecting the corks and they are coated with a little vaseline before inserting. Before using, the flasks

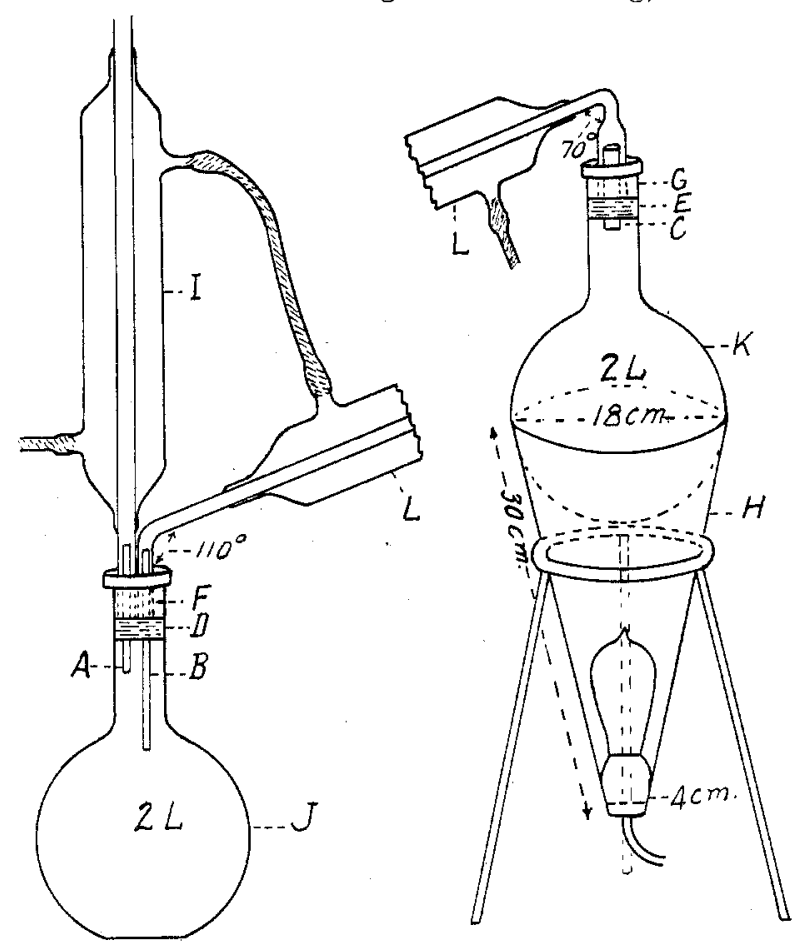

should be washed with ether to remove the excess vaseline. The glass tubes $A, B, C$ are about 5, 5, io $\mathrm{mm}$, respectively, in diameter. The refux condenser $I$ prevents the escape of ether.

To remcve the ether from the receiving flask $J$ lift up the condenser $I$ and insert a small siphon through $A$.

Okiahoma Agricultural, Experimeit Station STILLWATER

\title{
ADDRESSES
}

\section{A CENSUS OF THE AR'TIFICIAL DYESTUFFS USED IN THE UNITED STATES \\ By THOMAS H. NORTON}

The necessity for a complete enumeration of the artificial coloring matters, regularly consumed by the various manufac-

1 Address delivered before the American Chemical Society, at the New York Meeting, during the Symposium on "American Dyestuff Manufacture." turing industries of this country soon became evident when these branches were threatened in I9I4 by a dyestuff famine, as a result of the great European war.

Those who took into careful consideration the possibility of creating an independent American coal-tar dyestuff industry were obliged to study closely a number of factors bearing upon this exceedingly complicated question. Among these were such items as the supply of crude materials, the chemists and 


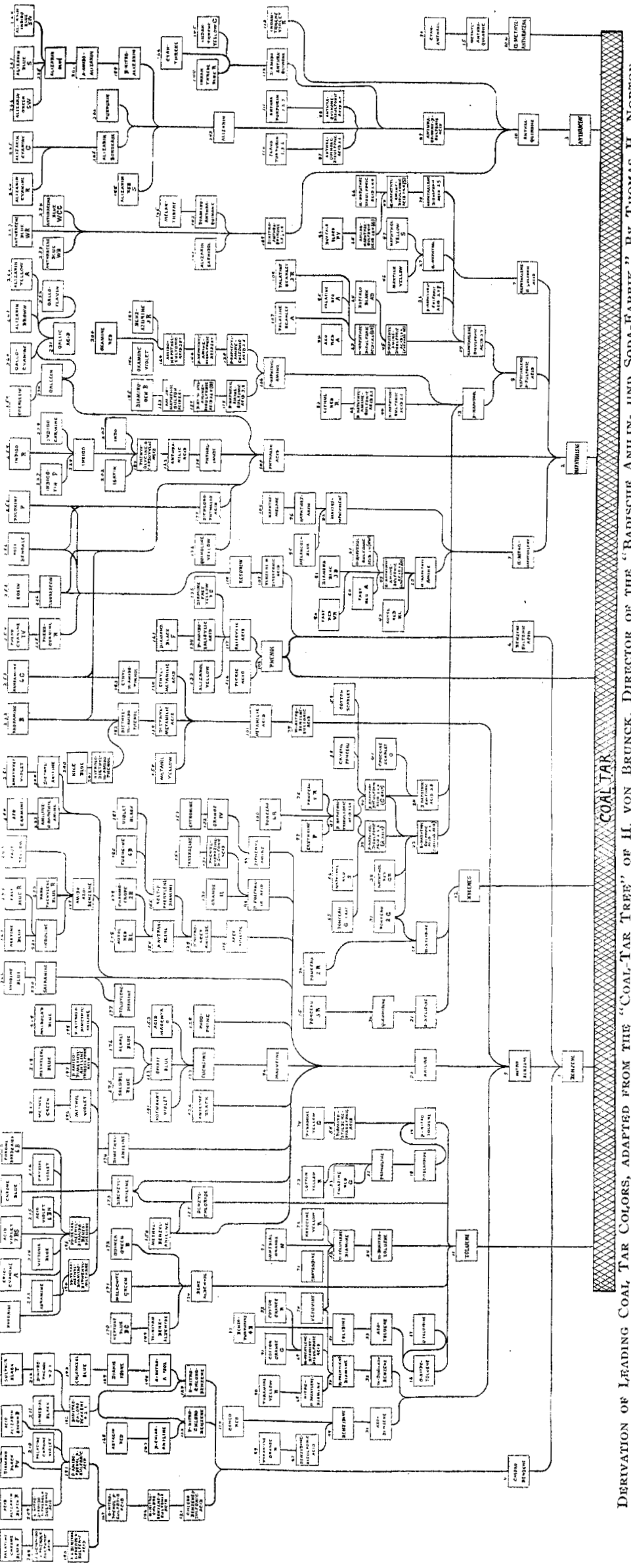

chemical engineers available, the probable attitude of the European interests, hitherto furnishing our synthetic dyes, upon the return of normal international conditions, the requisite fiscal and other legislation essential to safeguard American enterprise and capital against unfair competition on the part of such foreign rivals, etc.

First and foremost, however, came the factor of quantity. What is the total annual consumption of artificial colors in the United States? How many different dyes are in current use? What is the average annual consumption of each of these dyes?

The necessity of exact information on these three points is self-evident to some. For most, a brief explanation may be helpful.

In a general way we know how the great dyestuff industries of Germany and Switzerland are organized. We understand the relations of capital, of technical staff, ete., to output. From an economic standpoint it is necessary to know the total extent of the American market for this class of products, in order to estimate approximately the amount of capital required for a comprehensive industry, the number of trained chemists and engineers needed, and the quantities of coal-tar crudes to be provided. These form the main links in the chair connecting the gas works and the coke plant yielding coal tar and the gases laden with benzene and its homologues, with the multitude of mills and shops in which synthetic colors are employed to produce chromatic effects upon wares of the most varied nature-paper, textiles, leather, wood, ink, varnish, fur, feathers, foods, beverages, etc.

While such leading data are of prime importance from a general economic standpoint, of still greater value are the details concerning the specific products of the synthetic color industry.

\section{NECESSITY OF A CENSES OF DYES}

There are nearly I, 000 coal-tar dyestuffs of recognized standing in the tinctorial world; $i$. e., their chemical composition, or at least the methods of preparation, are publicly known. About twice as many are regularly manufactured, and enter into international trade, regarding the preparation or the composition of which little or nothing has been published. Many colors of both categories are encountered commercially in the form of several marks or brands. These represent slight modifications of the primary dye, sometimes in regard to shade, often in regard to convenience of application. The form in which a dye is prepared for use on cotton may not be the best form for the needs of the silk dyer. The requirements of the feather dyer may be quite different from those of the manufacturet of ink.

It is essential that the organizers of a national color industry know, with a certain approximation to accuracy, how much annually is consumed of each primary dye, and how much of each minor modification is employed. Without such data a manufacturer cannot calculate the size and number of the units to be constructed for the production of any given dye, and he is at an equal loss as to the equipment necessary to manufacture it in the different modifications of current use.

Again, the industry is one of great complexity, involving a high degree of coördination and of careful planning to avoid material loss in the way of byproducts. In the various steps intervening between 
a coal-tar "crude" and a finished dyestuff, each chemical reaction in the sequence is apt to produce certain percentages of closely allied compounds, isomeric substances as a rule. These latter may possess the same general chemical composition as the product more directly sought. The arrangement of the atoms in the molecule is, however, quite different. As a result, physical and chemical properties are totally unlike those characterizing the main substances. Such by-products possess, as a rule, distinct technical and commercial value. One may serve to make an entirely different dyestuff, another may be the raw material for manufacturing a valued medicinal; a third may be employed in the production of a photographic developer, etc.

It is evident, therefore, that the establishment of a synthetic color industry means an elaborate study of a multitude of interrelated operations, allied furthermore with numerous products in a group of closely connected industries, based likewise upon the use of coal-tar crudes. To some extent the changing whims of fashion enter into play. Back of every plan and calculation stands, however, the dominant factor of quantity.

It is now generally recognized that any intelligent effort to build up a comprehensive, self-contained, American coal-tar chemical industry must rest upon the solid foundations of accurate statistical data concerning the American market for artificial colors. In no other way can the creators of such an industry avoid duplication, overlapping, waste, and blundering, tentative struggles to adjust productive mechanism to a vague. indefinite demand. Without such fundamental data the future industry will be heavily handicapped by permanent overhead charges, accumulated as the result of being forced to feel its way in the dark, chemically, mechanically, and commercially.

If the coming American dyestuff industry is to hold its own successfully against foreign competition, it must be free from any unnecessary shackle. It must start into existence during these years of crimson-splashed struggle for Europe-of golden opportunity for this Republic-at the point where a brusque order to halt has been given the giant factories on the Rhine, the Main, and the Spree. It must utilize to the full all the gathered stores of experience accumulated during the six decades since Perkin's epochal discovery, and become a world factor in the seventh period of the history of synthetic color, at whose portal we now stand.

To no one is this country more indebted than to Dr. Bernhard C. Hesse, of New York, for a clear, forcible presentation of the complexity of the synthetic dyestuff industry and of the pressing necessity of accurate data on the domestic consumption of artificial colors. Attention should be directed to his briefer studies on the subject in the JOURNaL OF INDUSTrRIAL AND ENGINEERING ChEMISTRY, and to his more exhaustive paper read before the National Association of Cotton Manufacturers, in April, I915, and published in the Textile Manufacturers' Journal, May I, 1915, p. 60.

ACTION OF THE BUREAT OF FOREIGN AND DOMESTIC COMMERCE

Early in I 9 I 5 the embargo came into force, shutting off German dyes from this country. Long before, the relatively small supply of colors from England, France, Belgium and Holland had practically ceased and the somewhat more important source in Switzerland was threatened with paralysis.

The Bureau of Foreign and Domestic Commerce in Washington was following with the keenest interest, and even with anxiety, the initial steps taken bravely and resolutely by a small band of far-sighted American men, some manufacturers, some capitalists-all patriots-convinced that finally the opportunity had arrived to build up a genuinely national coal-tar chemical industry.

In the earnest desire to second their efforts and facilitate their plans, as well as to insure the most favorable and economical conditions for the rapid evolution of the new industry on a permanent basis, it was promptly recognized, in harmony with the reasoning detailed above, that nothing could be of such direct assistance as a "census" of the dyestuffs consumed normally in this country. Plans were carefully laid to carry out the work as expeditiously, accurately, and fully as the very limited appropriations at the command of the Bureau for such general purposes would permit.

\section{HOW THE CENSUS WAS TAKEN}

First of all it was necessary to decide upon the modus operandi. It was suggested by some, who had early recognized the desirability of such a "census," that the only available method for securing the needed data was to appeal to all consumers of artificial colors for their coöperation. It was thought that a ready response would be given to circular requests for detailed information regarding the annual consumption of coal-tar dyes by each user of the same. It was proposed, in order to overcome the customary repugnance of manufacturers to communicate facts of this nature, that the replies should be sent to some central financial institution, which would guarantee secrecy in collating the numerical information thus gathered.

A careful analysis of the problem showed that any such method of collecting data was impracticable. It would be impossible to secure a complete list of all users of dyestuffs in scores of trades and manufacturing branches. Assuming that figures could be obtained from all users of colors, their compilation would be a herculean task. Suppose that 5 tons of Congo Red are consumed annually in this country. This amount might be divided up among several thousand consumers, in lots ranging from 5 to roo pounds.

With a somewhat elementary knowledge of human psychology, it was furthermore certain that no replies could be expected from the great majority of the recipients of circular requests. Indifference, suspicion, or pure laziness are serious factors to overcome.

\section{BRITISH ATTEMPT TO TAKE A CENSUS OF COLORS}

The correctness of this conclusion has recently been abundantly verified by trans-Atlantic experience. British textile and allied interests have been forced to deal with a far more serious "dyestuff famine" than has been the case in the United States. There was a șimilar determination to build up a genuinely national color industry. The necessity of a dyestuff "census" was likewise recognized as of paramount importance. An influential committee, representing makers and consumers of dyes, took the matter in hand. Appreciating the futility of dealing directly with the multitude of individual users of colors, the committee decided to collect its statistics through the various powerful organizations of trades employing large quantities of dyestuffs and then double the results, thus roughly approximating at the entire national consumption of the various colors. After months of labor the committee was forced, in November, I $_{95} 5$, to report a practical fiasco. Replies were secured from but ig associations or large individual consumers. The figures obtained covered but 3,145 short tons, perhaps I2 per cent of the national consumption.

\section{FEATURES AND VALUE OF THE CENSUS}

The method adopted by the Bureau of Foreign and Domestic Commerce was much more simple, direct and accurate. As in the case of Great Britain, nearly nine-tenths of the normal American consumption is derived from European sources. It was decided to use the data based upon the imports of artificial colors into this country during the 12 months ending June 30, 1914-a month before the outbreak of the present war. The remaining tenth is covered by the returns of the Burean of the Census for the domestic coal-tar dyestuff industry based upon the production of the calendar year 19I4. No serious 
SLMMARY OF THE MOST IMFORTANT COLORS IMPORTED BY U. S. DURING FISCAL YEAR 1913-14

The abbreviation V.M. denotes "various marks." The serial numbers employed correspond to those found in Schultz's "Farbstofftabellen" (edition of 1914). Numbers preceded by letters refer to colors regarding the manufacture and chemical composition of which little or nothing is known. Azo

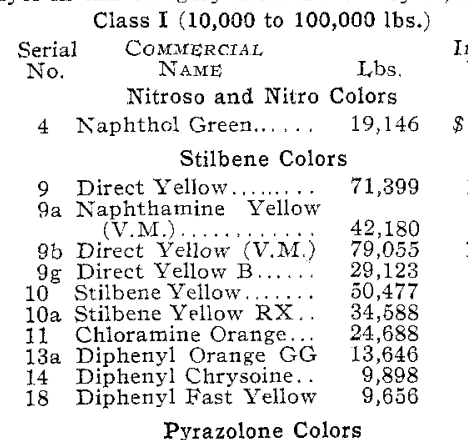

19 Fast Light Yellow.... 33,514

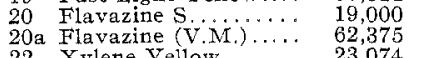
22 Xylene Yellow........ Azo Colors

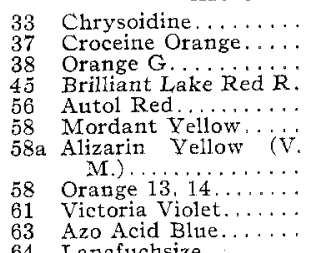
64 Lanafuchsize....... 70 Brilliant Orange $O$..... 73 Felio Fast Red..... 73a Iithol Fast Scarlet...' 80a Wool Scarlet (V.M.) 82a Ponceau Anth acen Brown (V,M.).... 96a Chrome Fast Yellow. 96a Chrome Fast Yellow. 102 Diamond Fla 112 a Claret R.ed. 118 Geranine............. 132 I ake Red P... 137 Acid Yellow.

139 Orange IV.

140 Carcumeine.

141 Azo Yellow. . .

141b Indian Yellow V.M. 146 Azo Fuchsine G..... 147 Azo Fuchsine

152 Prange $R$ O,$\ldots 34$ 152a Permanent Red (V. 104 Palatine Chrom 159 a Vigouretux Fast Black 160 Fast Brown 1 . 161 Fast Red A. 163 a Carmoisine (V.M.) 1 . 164a Diamond Blue R 168 Amaranth.

168 b Wool Red (V.M) 169 Cochineal Red. 173a Lithol Red (V.M.).
177 Lordant Yellow... 777 a Anthracene Yellow 180 Erichrome Blue Black 181 Salicine Black $\quad$ C................. 184 Eriochrome Black A.
185 Anthracene Chrome 188 Sulfone Acid Blue $R$ 189 Sulfone Acid Blue B. 198 Thiazine Yellow. 212 a Acid Brown (V.M.) 217 Agalma Black $10 \mathrm{~B}$ 217 c Naphthol Blue Black 217 e Acid Black (VM.). $217 \mathrm{~g}$ Wool Black (V.M.) 217 h Acid Wool Black. 236 Wool Red. 247 Scarlet.... 257b Tolyl Blue ............... 269 Acid Black .......... 272 Brilliant Black..... $272 b$ Wool Black (V.M.).j.
$275 a$ Chrome Black (V.M.)
Serial Commercral

$27 \mathrm{c}$ Chrome Fast Black

277 Anthracene ..... Acid

279 Black ............

283 Bismarck Brown.....

296 Cotton Yellow.........

303 Renol Brilliant Yellow

307 Congo.

312 Congo Corinth.......

313 Congo Rubine.

319 Diamine Scarlet...

326 Oxy Diamine Violet.

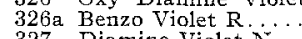
327 Diamine Violet $\mathrm{N}$ 333d Develop Black...... 334 Diphenyl Blue Black 335 Naphthamine Black. 337 Benzo Blue...................... 338 Naphtbarnire Bli1e.. 343 Diamine Fast Red... 344 Diamine Browt1..... 348 Diphenyl Brown BN. 358 Diphenyl Red........ 360 Pyramine Orange $R$. 365 Benzopurpurine $(\mathrm{V}$. 366 Deltapurpurine $\overline{5}$ В. 370 Brilliant Congo.$\cdots$ 384 a Diamine Blue (V.M ) 392 Toltylene Orange... 400 Acid Anthracene Red. 405 Benzopurpurine $10 \mathrm{~B}$ 410 Benzazurine (V.M.) 416 Brilliant Azurine $5 \mathrm{G}$.
418 D i a m i n e Brilliant 419 Chicago Blue $\mathrm{R}$ W. 419 Cxicago Blue R W 421 Oxamine Blue B.... 426 Benzamine Pure Blu 428 a Direct Blue (V.M.). 449 Tristlfon Brown.. 456
456 a Benzo Fast Blue (V 462 Direct Deep Black 462 C Cotton Black (V.M.). 462 d Union Black (V.M.) 469 Chloramine Black.
469 a Chloramine Black 474 Oxamine Green B... 474a Diamine Green (V. 476a Benzamine Brown 3 477a Naphthamine Brown 478 Columbia Green ... 478a Direct Green (V.M.) 19,313 $\begin{array}{ll}485 a & \text { Benzo Brown (V.M.); } \\ 41,905 \\ 23,975\end{array}$ Unclassified Azo Colors

A 6 Chrome Fast Black A 12 Columbia Brown (V) A 16 Columbia Fast Blue A 28 Naphthogene Blue $(V$. A 32 verol $\left(\mathrm{M} . \mathrm{M}^{2} \ldots \ldots\right.$ A 44 Colamine Blue B.... A 69 Corvan Black (V.M.) A 81 Lithol Fast Orange R A 88 Oxamize Black (V.M.) A102 Oxamine Copper Blue A104 Oxamine Dark Biue A108 Oxamine Dark Brown A122 Palatine Chrome Biue A124 Palatine Chrome Green G....... A131 Scarlet (V.M.) W. ${ }^{\text {A142 }}$. A144 Acid Black E, M. . A150 Acid Silk Black R... A157 Benzo Chrome Black A166 Benzo Dark Green B;

\begin{tabular}{|c|c|}
\hline Lbs. & $\begin{array}{l}\text { Invoice } \\
\text { Value }\end{array}$ \\
\hline 35,999 & $\$ 10,532$ \\
\hline $\begin{array}{l}17,793 \\
36,674 \\
27,576\end{array}$ & $\begin{array}{l}2,647 \\
9,010 \\
5,352\end{array}$ \\
\hline $\begin{array}{l}18,985 \\
21,437 \\
12,786 \\
12,040 \\
62,854 \\
39,748 \\
46,113 \\
28,887 \\
11,514 \\
12,467 \\
13,107 \\
17,495 \\
26,240 \\
47,969 \\
19,035 \\
11,707 \\
47,724 \\
63,716 \\
11,636 \\
13,471 \\
12,808 \\
21,329 \\
19,905\end{array}$ & $\begin{array}{r}1,607 \\
6,161 \\
3,290 \\
1,687 \\
8,257 \\
6,030 \\
6,329 \\
9,027 \\
1,938 \\
1,552 \\
2,840 \\
4,333 \\
4,415 \\
7,132 \\
789 \\
2,455 \\
17,131 \\
12,457 \\
2,568 \\
4,015 \\
5,001 \\
7,818 \\
4,223\end{array}$ \\
\hline $\begin{array}{l}21,090 \\
20,284 \\
19,133 \\
21,725 \\
55,562 \\
17,560 \\
47,708 \\
78,699 \\
18,395\end{array}$ & $\begin{array}{r}1,442 \\
3,646 \\
3,133 \\
3,687 \\
13,236 \\
5,174 \\
1,1,181 \\
21,018 \\
3,206\end{array}$ \\
\hline $\begin{array}{l}11,592 \\
10,176 \\
14,091 \\
21,800 \\
12,881 \\
21,322 \\
16,781 \\
73,936\end{array}$ & $\begin{array}{r}2,496 \\
3,364 \\
2,436 \\
3,749 \\
5,663 \\
5,366 \\
5,255 \\
20,607\end{array}$ \\
\hline 26,559 & 8,439 \\
\hline $\begin{array}{l}32,830 \\
91,485 \\
61,218 \\
20,095\end{array}$ & $\begin{array}{r}5,032 \\
22,206 \\
9,044 \\
5,278\end{array}$ \\
\hline $\begin{array}{l}19,505 \\
23,832\end{array}$ & $\begin{array}{l}3,951 \\
5,134\end{array}$ \\
\hline 53,268 & 8,318 \\
\hline 16,988 & 2,470 \\
\hline $\begin{array}{l}48,734 \\
24,749 \\
19,313 \\
41,905 \\
23,975\end{array}$ & $\begin{array}{l}9,452 \\
4,723 \\
4,291 \\
7,125 \\
5,207\end{array}$ \\
\hline
\end{tabular}

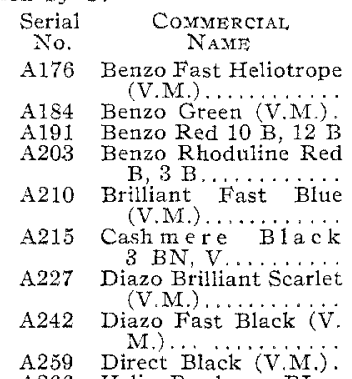

A259 Direct Black (V.M.).

A277 Orange RO.......

A286 Pluto Black (VM)

A292 Pluto Brown (V.M.)

A336 Azo Wool violet (V.

A346 Diamine Catechine ( $V$

A351 Diamine Fast "Blue

A355 Diamine Fast Orange

A35s Diamine Fast Orange

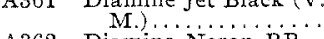

A362 Diamine Neron BB

A368 Diamine Sky Blue FF

A384 Oxy Diamine Brown

A385 Oxy Diamine Carbon

A388 Para Diamine Black

A403 Salicine Biue B.......

$A \notin 14$ Amido Naphthol

A418 Azo Acid Black (V)

A430 Fast Mordant Blue B;

A437 Naphthalene Biue B,

A439 Victoria scarle $R$,

A444 Direet Green (V.M.).

A451 Heligoland Black

A469 Oxychrome Brown (

A472 Oxychrome Yellow (V.

A478 Triazol Blue (V.M.)

A485 Triazol Brown

A515 Brilliant Scarlet 2 R,

$4 \mathrm{R} . . . . . . . . .$.
A524 Anthracy

A527 Croceine Scarlet Mì.

A531 Acid Blue Black......

$76,451 \quad 10,172$

A541 Diazogene Black (V.

A550 Direct Black ABC,

A552 Direct Chrome Brown

A552 Drazaline Blue (V.M.)

A566 Drazaline Brown (V.

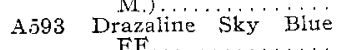

A600 Excelsior Black.

A603 Hydrazol Black......

A605 Hydrazol Chrome

Black CB, DB....
A612 Chicago Red III....

A617 Diphenyl Blue (V.M.)

A622 Diphenyl Deep Black
A629 Diphenyl Green (V.

A629 Diphenyl Green (V.

Ibs Invoice

$13,018 \$ 5,541$

$\begin{array}{lr}13,506 & \$, 540 \\ 16,502 & 2,850 \\ 19,420 & 4,715\end{array}$

$11,873 \quad 1,813$

$11,553 \quad 3,309$

$12,269 \quad 1,881$

$38,909 \quad 14,210$

$29,330 \quad 7,476$

$\begin{array}{rr}12,048 & 2,019 \\ 14,703 & 793 \\ 24,288 & 2,246\end{array}$

$14,066 \quad 1,619$

$\begin{array}{ll}30,010 & 6,034 \\ 14,580 & 2,542\end{array}$

$30,189 \quad 3,124$

$12,944 \quad 3,298$

$66,876 \quad 14,942$

$28,880 \quad 7,227$

$17,387 \quad 4,819$

$\begin{array}{ll}14,091 & 4,315 \\ 36,982 & 6,204\end{array}$

$\begin{array}{ll}17,068 & 2,851 \\ 41,115 & 7,574\end{array}$

$23,498 \quad 3,810$

$34,388 \quad 7,864$

$18,634 \quad 2,690$

$10,750 \quad 1,210$

$19,500 \quad 3,042$

$17,000 \quad 4,612$

$28,000 \quad 5,102$

$22,400 \quad 2,379$

$25,132 \quad 4,151$

$10,490 \quad 2,235$

$10,085 \quad 1.985$

$\begin{array}{ll}10,148 & 1,580 \\ 17,067 & 2,854\end{array}$

$19,489 \quad 2,647$

$12,565 \quad 1,425$

$24,979 \quad 6,385$

$12,210 \quad 2,235$

$12,952 \quad 4,365$

$30,042 \quad 6,813$

$15,245 \quad 2,804$

4,425

$21,756 \quad 4,979$

$10,940 \quad 5,204$

$\begin{array}{rr}59,956 & 16,690 \\ 10,981 & 1,629\end{array}$

$51,694 \quad 7,499$

$\begin{array}{ll}13,195 & 2,420 \\ 12,677 & 3,842\end{array}$

$21,098 \quad 4,216$

A664 Chlorantine Brown
(V.M.) ............

$18,021 \quad 4,667$

AB82 (V.M.) ..............

$18,267 \quad 4,034$

6,452

A682 Chrome Fast Green

A687 Cupranil Brown ( $\dot{V}$.

$12,204 \quad 3,550$

$12,780 \quad 1,41$ 
SUMMARY OF THE MOST IMPORTANT COIORS IMPORTED BY U. S. DURING FISCAL YEAR 1913-14-(continued)

\begin{tabular}{|c|c|c|c|c|c|c|c|c|c|c|c|}
\hline $\begin{array}{l}\text { trial } \\
\text { so. }\end{array}$ & $\begin{array}{l}\text { COMMERCIAL } \\
\text { NAME } \\
\text { Triphenyimethane }\end{array}$ & $\begin{array}{l}\text { Lbs. } \\
\text { Colors }\end{array}$ & $\begin{array}{l}\text { Invoice } \\
\text { Value }\end{array}$ & $\begin{array}{l}\text { Serial } \\
\text { No. } \\
739\end{array}$ & $\begin{array}{l}\text { COMMERCIAL. } \\
\text { NAME } \\
\text { medial Maroon } \mathrm{B} \\
\text { tigene Green }\end{array}$ & $\begin{array}{l}\text { Lbs. } \\
15,496\end{array}$ & $\begin{array}{l}\text { Invoice } \\
\text { Value } \\
\$ 2,885\end{array}$ & $\begin{array}{l}\text { Serial } \\
\text { No. }\end{array}$ & $\begin{array}{c}\text { COMMERCIAI } \\
\text { NAME } \\
\text { Unclassified Coal-? }\end{array}$ & $\begin{array}{l}\text { Lbs. } \\
\text { Colors }\end{array}$ & $\begin{array}{l}\text { nvoice } \\
\text { Value }\end{array}$ \\
\hline $\begin{array}{l}497 \mathrm{a} \\
499\end{array}$ & 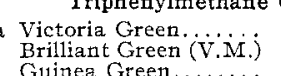 & $\begin{array}{r}\mathbf{4} 100 \mathrm{rs} \\
\mathbf{4}, 595 \\
73,904 \\
14,666\end{array}$ & $\begin{array}{r}\$ 10,3 \\
16,3 \\
3,3\end{array}$ & 746 & $\begin{array}{l}\text { Katigen Green (V. } \\
\text { M.)........... } \\
\text { Kryogere Brown } \mathrm{G}\end{array}$ & $\begin{array}{l}63,929 \\
10,313\end{array}$ & $\begin{array}{r}9,950 \\
972\end{array}$ & & & & \\
\hline $\begin{array}{l}502 \\
502 a \\
-02 a\end{array}$ & $\begin{array}{l}\text { Guine } \\
\text { Acid }\end{array}$ & & & & Unclassifed Sulfur & Colors & & & & & \\
\hline 503 & $\begin{array}{l}\text { Neptu } \\
\text { M.) }\end{array}$ & 40,868 & 13,825 & $\begin{array}{lr}\mathrm{S} & 3 \\
\mathrm{~S} & 12\end{array}$ & $\underset{\text { Sulfur }}{\text { Sulfur }}$ & $\begin{array}{l}3,434 \\
9,691\end{array}$ & $\begin{array}{r}15,489 \\
9,505\end{array}$ & 20 & $\begin{array}{l}\text { uinea Bordeaux } \\
\text { M.) , . . . . }\end{array}$ & & \\
\hline 505 & $\begin{array}{r}\text { Light } \\
\text { ish) }\end{array}$ & 24,9 & 5,960 & $\begin{array}{l}\text { S } 18 \\
\text { S } 26\end{array}$ & $\begin{array}{l}\text { Sulfi } \\
\text { Sulfi }\end{array}$ & & 5, & $\begin{array}{l}24 \\
27\end{array}$ & $\begin{array}{l}\text { Ind } \\
\text { Met }\end{array}$ & & \\
\hline $\begin{array}{l}505 a \\
506\end{array}$ & $\begin{array}{l}\text { Acid Green (V.M.) } \\
\text { Erioglaucine (V.M.) }\end{array}$ & $\begin{array}{l}46,461 \\
66,526\end{array}$ & $\begin{array}{l}20,176 \\
28,971\end{array}$ & S 37 & $\begin{array}{l}\text { Suthur lind1 } \\
\text { Katigene B } \\
\text { (V.M.)... }\end{array}$ & 006 & 336 & 31 & me Brown & 14,301 & 3,99 \\
\hline 512 & $\begin{array}{l}\text { Magenta } \\
\text { Crystal Violet.................... }\end{array}$ & $\begin{array}{l}87,102 \\
33,653\end{array}$ & $\begin{array}{l}25,659 \\
13,664\end{array}$ & S 45 & $\begin{array}{l}\text { Katgene Brown } 2 \text { R, } \\
\text { V.... }\end{array}$ & 811 & & & & $\begin{array}{l}57,313 \\
13,344\end{array}$ & $\begin{array}{l}7,27 \\
1,24\end{array}$ \\
\hline $516 a$ & $\begin{array}{l}\text { Violet (V.M.) } \\
\text { Benzyl Violet. }\end{array}$ & $\begin{array}{l}18,219 \\
22,387\end{array}$ & $\begin{array}{l}5,289 \\
6,018\end{array}$ & s 49 & Katigene Direct Blue, & 11299 & 452 & & t Blue 4 & 23,585 & 5,19 \\
\hline & $\begin{array}{l}\text { Ethyl Pur } \\
\text { Aniline B1 }\end{array}$ & $\begin{array}{l}51,933 \\
50,563\end{array}$ & $\begin{array}{l}23,101 \\
18,586\end{array}$ & S 51 & Katigene Indigo (V. & 4215,20 & & U 86 & Columbia Fast Black & 82040 & 0,10 \\
\hline & Acid I & $\begin{array}{l}19,098 \\
13,078\end{array}$ & $\begin{array}{r}4,030 \\
4,362\end{array}$ & $S_{S}^{S} 55$ & Katigene Khaji $\mathrm{G} \ldots$ & $\begin{array}{l}42,157 \\
14,242\end{array}$ & $\begin{array}{l}5,924 \\
1,691\end{array}$ & $\tau 100$ & Basic Kraft Brown & 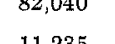 & 15,75 \\
\hline 527 a & $\begin{array}{l}\text { Acid Violet } \\
\text { Fast Acid Violet }\end{array}$ & $\begin{array}{l}16,106 \\
12,919\end{array}$ & $\begin{array}{l}5,360 \\
3,229\end{array}$ & & rown & 68,864 & 9,386 & $\mathrm{U} 109$ & Brilliant Scarlet $(v$. & 1,235 & 2,03 \\
\hline 30 & Acid Violet. & $\begin{array}{l}12,0,50 \\
65,395\end{array}$ & $\begin{array}{r}12,806 \\
20,054\end{array}$ & S 62 & $\begin{array}{c}\text { Katigene Ye } \\
\text { GG, GR... }\end{array}$ & 5,227 & 318 & 0121 & $\begin{array}{r}\mathrm{M} \\
\text { Corv }\end{array}$ & 32 & $\begin{array}{l}2,58 \\
3,24\end{array}$ \\
\hline & $\begin{array}{l}\text { Acid Violet (V.M.) } \\
\text { o Formyl Violet (V.M.) }\end{array}$ & $\begin{array}{l}03,0 \\
19,8\end{array}$ & $\begin{array}{r}20,9 \\
4,1\end{array}$ & S 65 & $\underset{\text { Bro }}{\text { Katig }}$ & 6,826 & & & & & \\
\hline & $\begin{array}{l}\text { Guinea Vi } \\
\text { B....... }\end{array}$ & 18,8 & 5,1 & S 75 & $\begin{array}{l}\text { Immedial Direct Blue } \\
\text { (V.M.) }\end{array}$ & 820 & 145 & $\begin{array}{l}\text { U145 } \\
\text { U151 }\end{array}$ & $\begin{array}{l}\text { Jap } \\
\text { Jet }\end{array}$ & & \\
\hline & 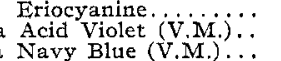 & $\begin{array}{l}25,091 \\
19,960 \\
31,499\end{array}$ & $\begin{array}{r}11,987 \\
6,310 \\
6,275\end{array}$ & s 76 & Ial Ind & 90,077 & 13,141 & $\begin{array}{l}\mathrm{U} 155 \\
\mathrm{U} 158 \\
\mathrm{~T} 163\end{array}$ & LBO, & & $\begin{array}{r}10,218 \\
4,843\end{array}$ \\
\hline $538 a$ & $\begin{array}{l}\text { Cot } \\
\text { Solu }\end{array}$ & $\begin{array}{l}45,019 \\
86,523\end{array}$ & $\begin{array}{r}9,809 \\
31,093\end{array}$ & S 78 & $\operatorname{Imn} \mathrm{G}$ & , & 10,6 & & H & & \\
\hline $\begin{array}{l}543 c \\
545\end{array}$ & $\begin{array}{l}\text { Acic } \\
\text { Pate }\end{array}$ & $\begin{array}{l}14,467 \\
40,848\end{array}$ & $\begin{array}{r}4,916 \\
10,229\end{array}$ & $\begin{array}{l}\text { S } 84 \\
\text { S } 86\end{array}$ & i.j & & & $\begin{array}{l}80 \\
83\end{array}$ & $\begin{array}{l}P_{i} \\
Q u\end{array}$ & & \\
\hline $545 \mathrm{a}-1$ & Blue $(\dot{V}, \dot{M})$ & & & $\begin{array}{l}\text { S } 97 \\
\$ 100\end{array}$ & $\begin{array}{l}\text { Thiogene Blu } \\
\text { Thiogene } \mathrm{Br}\end{array}$ & & & & & & 42 \\
\hline & Brillian & & & & & 97,551 & 10,601 & U206 & & & \\
\hline 46 & $\begin{array}{l}\text { Cyanc } \\
\text { Eriock }\end{array}$ & & & S155 & Pyr & & & 17 & & & \\
\hline & $\mathrm{BC}$ & 21,070 & 14,480 & $168>>2>$ & Sulf & 63,4 & 6,6 & $\mathrm{U}^{2}$ & & & \\
\hline & Diphe & hame & & & Cro & & & & & & \\
\hline 564 & Nap & $\frac{2}{3}+2>-2$ & 5,9 & & Anthraquinone and $A$ & Allied $\mathrm{C}$ & & $\mathrm{U} 279$ & Brili & & \\
\hline & Cyan & $\begin{array}{l}50 \\
10\end{array}$ & $\begin{array}{r}10, \\
2,\end{array}$ & 760 & $01 d$ & & & U283 & 'ivi. & 11,289 & 75 \\
\hline & Color & & & 761 & $\because 1 \mathrm{~d}$ & & 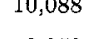 & & & $\begin{array}{l}41,082 \\
11,784\end{array}$ & 66 \\
\hline 57 & Rhod & 3 & 18,4 & $7633+2>3$ & & 50,496 & 2,052 & $\mathrm{U} 293$ & & & \\
\hline & & & & 105 & & & & & & & \\
\hline & tiv. & & & & Feen $B$ & & & & & & \\
\hline 587 & & 3 & $\begin{array}{l}13,4 \\
13,1\end{array}$ & & & 68,419 & 21,516 & & $\mathrm{Co}+\mathrm{C}_{2}$ & & \\
\hline & $\begin{array}{l}\text { Eos } \\
\text { Bro }\end{array}$ & & & $768 a$ & Indanth & & & $\mathrm{U} 333$ & $j$ & & 3,116 \\
\hline & & & & 774 & & & & & & & \\
\hline $590 \mathrm{a}$ & Acic & $\begin{array}{l}17,48 \\
15,40\end{array}$ & $\begin{array}{l}7,388 \\
8,817\end{array}$ & & Aliz & 14,23 & & & Dit & & 4,799 \\
\hline & lor & & & & & & & & & & 10,67 \\
\hline & Pat & & & & & & & 85 & & & $\begin{array}{l}10 \\
36 \\
36\end{array}$ \\
\hline & $\frac{L}{R}$ & & 8,2 & & Anth & 22,444 & 7,174 & $\begin{array}{l}0391 \\
-202\end{array}$ & & & \\
\hline & & & 0,0 & 800 & WB, & & & & & & 2 \\
\hline & & & $\begin{array}{r}5,8 \\
13,4\end{array}$ & & & 54 & 9,2 & & & & 18 \\
\hline & $\mathrm{Cu}_{\mathrm{u}}$ & ten & & & & & 69 & & $\bar{U}$ & & \\
\hline 312 & 8 & & & & & & & & e Blue & & \\
\hline & & 79,5 & 28,1 & & & & & U440 & flow G, 5 G, & & \\
\hline & $\begin{array}{c}x \text { e } \\
\text { Iuble) } \ldots \mathrm{w}\end{array}$ & & & & & & & & & & \\
\hline $\begin{array}{l}616 \\
617\end{array}$ & $\begin{array}{l}\text { Pri } \\
\text { Col }\end{array}$ & & & & $\begin{array}{l}\text { Aliz } \\
\text { Heli }\end{array}$ & & 6,954 & & & & \\
\hline & & 86,0 & 10,1 & & Algo & & & & & & \\
\hline & 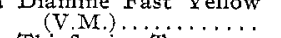 & 8 & 1 & 827 & Ind & & & & & & \\
\hline & Th & & & & $\mathrm{P}$ & & & & & & 4,78 \\
\hline & tiazine & & & & & & & & B, & & \\
\hline 627 & $\begin{array}{l}\mathrm{Gal} \\
\mathrm{Cot}\end{array}$ & & $\begin{array}{r}27,2 \\
9,6\end{array}$ & & & 10,163 & 4,284 & $\begin{array}{l}\tau 610 \\
\tau 682\end{array}$ & & & \\
\hline & $\mathrm{Met}$ & & & & ......... & & , 3 & & Black & & \\
\hline 661 & & 18 & 7, & 849 & Inde & & & & & & 27 \\
\hline & & & 12 & 850 & Bi u & & & & & & \\
\hline & Indochr & & & & $\ldots$ & 31,6 & 4,272 & & (1/2) & & 31 \\
\hline & lors & & & & & & & 711 & & & 3,01 \\
\hline 672 & $\begin{array}{l}\mathrm{Az} \\
\mathrm{Sa}\end{array}$ & $\begin{array}{l}17,5 \\
59,9\end{array}$ & $\begin{array}{r}5,45 \\
21,27\end{array}$ & & & & & U716 & $\mathrm{JC}, 6$ & & \\
\hline & it Gray (V. & & & & B, 3 в & & & 31 & & & \\
\hline 69 & Ind & & & & Indigo and $\mathrm{I}$ & & & & I. $\ldots \ldots$ & & \\
\hline 699 & Inc & & & & & & & & 20 & & \\
\hline a & $\begin{array}{l}\text { Water } \\
\text { Indocyanin }\end{array}$ & $\begin{array}{l}21,77 \\
23,13\end{array}$ & $\begin{array}{l}5,5 \\
5,2\end{array}$ & $\begin{array}{l}79 \\
81\end{array}$ & & & 7 & $\begin{array}{l}34 \\
48\end{array}$ & & & i1 \\
\hline & lors & & & & & & & 145 & & & 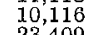 \\
\hline & Sul & & & & in & & & & & & \\
\hline 710 & ai Yellow i & & & & & & & & & & \\
\hline 72 & ial Brown $(\dot{v}$. & 13, & & & Helindone Pink (V. & & & & & & \\
\hline & M.j) & & & & e Red 3 B.. & $\begin{array}{l}14,48 \\
2787\end{array}$ & 0,942 & $220 \mathrm{~b}$ & $\because x+3$ & & 16 \\
\hline & Indigo (V. & & & & $\therefore$ & & & & $\ldots .$. & & \\
\hline & & & & & & & & & ack & & \\
\hline
\end{tabular}




\begin{tabular}{|c|c|c|c|c|c|c|c|c|c|c|c|}
\hline \multirow{3}{*}{$\begin{array}{l}\text { Serial } \\
\text { No. } \\
269 \text { a I }\end{array}$} & \multicolumn{2}{|l|}{ JMMARY OF } & & \multicolumn{2}{|l|}{ RS 1MPORTL } & & \multicolumn{4}{|c|}{ YEAR 1913-14- } \\
\hline & $\begin{array}{l}\text { COMMERCIAL } \\
\text { NAME }\end{array}$ & I bs. & $\begin{array}{l}\text { Invoice } \\
\text { Value }\end{array}$ & $\begin{array}{l}\text { Serial } \\
\text { No. }\end{array}$ & $\begin{array}{l}\text { CoMmERcial } \\
\text { NAME }\end{array}$ & I bs. & $\begin{array}{l}\text { Invoice } \\
\text { Value }\end{array}$ & $\begin{array}{l}\text { Serial } \\
\text { No. }\end{array}$ & $\begin{array}{l}\text { CoMmerctaL } \\
\text { NAME }\end{array}$ & $\mathrm{L}, \mathrm{b}$ & $\begin{array}{l}\text { Invoice } \\
\text { Value }\end{array}$ \\
\hline & $\begin{array}{l}\text { Naphthol Black (V. } \\
\text { M.) } \ldots \ldots \ldots \ldots \ldots \text {. }\end{array}$ & & & 774 & $\begin{array}{l}\text { Alizarin Black S, SR, } \\
\text { NR } \ldots \ldots \ldots \ldots \ldots\end{array}$ & & & $\begin{array}{l}778 \\
806 a\end{array}$ & $\begin{array}{l}\text { Alizarin (Synthetic) } \\
\text { Alizarin Black (V.M.) }\end{array}$ & $\begin{array}{l}202,392 \\
229,500\end{array}$ & $\begin{array}{r}\$ 20,465 \\
33,275\end{array}$ \\
\hline $\begin{array}{l}284 \\
304\end{array}$ & $\begin{array}{l}\text { Bismarck Bro } \\
\text { Chrysophenin }\end{array}$ & $\begin{array}{l}170 \\
148\end{array}$ & & $\begin{array}{l}782 \\
789\end{array}$ & $\begin{array}{l}\text { n Brown ( } \mathrm{V} \cdot \mathrm{M} \text { ) } \\
\text { cene Blue WR. }\end{array}$ & & & & Class IV $(300,000$ to 4 & $400,000 \mathrm{lbs}$ & \\
\hline $333 \mathrm{~b}$ & $\mathrm{Dia}$ & & & 80 & & & & & & & \\
\hline 424 & o Blue & & & $808 a$ & $\left.\dot{M}_{1}\right)$ & & & 27 & & & \\
\hline $436 \mathrm{a}$ & Black.; & & & 838 & & & & 2 & & & \\
\hline $\begin{array}{l}445 \mathrm{a} \\
455 \mathrm{a}\end{array}$ & bia Black (V. & & & $C 3$ & Wo & & & 363 & & & \\
\hline & $\stackrel{\mathrm{M}}{\mathrm{M}}$ & & & 7 & DIo & & & $\mathrm{A} 432$ & ed & & \\
\hline & Dent & 100 & & & 30 & 0 & & $70+2>3$ & Sol. & & \\
\hline & (V.M.). & 146.629 & & & & & & $803 a$ & Al & & \\
\hline A 387 & Oxy Diaminogen & & & 23 & & & & & Class V $(400,000$ to 50 & $00, \mathrm{C}$ & \\
\hline & & 139 & & 134 & $M$ & & & 33 & 0 & & \\
\hline $\begin{array}{l}495 \\
543\end{array}$ & $\begin{array}{l}\mathrm{Ma} \\
\mathrm{Pat}\end{array}$ & & & $\begin{array}{l}157 \\
173\end{array}$ & & & & 49 & & & \\
\hline & Vic & & & & & & & 842 & ne Blue & 180 & 169,780 \\
\hline $\begin{array}{l}565 a \\
606\end{array}$ & $\begin{array}{l}\text { Wo } \\
\text { Phe }\end{array}$ & & & $303 a$ & $\stackrel{\mathrm{Pa}}{\mathrm{Col}}$ & & & & I 500, & 0 & \\
\hline & $\mathrm{Bl} 11$ & & & & & & & $2 \mathrm{a}$ & & & \\
\hline & & $78 \overline{7}+20$ & & & & & & & $Z$ & & \\
\hline
\end{tabular}

interference in the output of American colors occurred until after the beginning of 1915 .

With the cordial coopperation of the Secretary of the Treasury, all the invoices for the year in question were sent by the collectors of customs at the various ports of entry to a central point, where the essential data were transcribed. These include weight, value and price. Some 37,500 different transcripts, each covering these three items, were necessary.

These entries are found under 5,674 heads, each representing a distinct commercial designation. It must not be inferred, however, that this number of different colors comes into consideration. Many standard dyes are manufactured by several firms in the same country as well as in various countries. Frequently, some or all of the competing manufacturers use entirely different trade names for identical wares.

Thus, the red color, known chemically as sodium $\alpha$-naphthalene azo- $\alpha$-naphthol-disulfonate, is manufactured under the name of Palatine Red by the Badische Co. The Bayer Co. sells it under the name of Naphthorubine. Primuline is encountered commercially as Polychromine, Thiochromogen, Aureoline and Sulfine. Malachite Green, a favorite color, is found under 38 different designations, few representing even slight variations in the exact chemical composition.

The reduction of this extensive vocabulary to the limits of the list given in the census has required highly specialized editing. It is hoped that the arrangement and the full use of synonyms are such as to render the published results of the greatest utility, not only to all engaged in the manufacture of artificial dyestuffs, and especially in plannit1g for the establishment of a comprehensive American color industry, but also to all dealers in the wares and to all consumers of dyeing materials.

All three of these categories have hitherto been indebted to the painstaking labors of several prominent German color chemists, notably of Gustav Schultz and Paul Julius, for complete and detailed classifications of the coal-tar dyes in current use. The carefully elaborated "Farbstofftabellen," devised by the two authors, reached a fifth edition in 19r4. These "tables," divided into groups according to chemical relationship, give for every artificial dye of known composition or preparation the commercial designation, the scientific name, the chemical formula, the most advantageous process for technical production. physical and chemical properties, methods of application, tests and full references to patents and literature. They have for years been the vade mecum of all connected with the manufacture of colors, their commerce, and their manifold uses.

It has remained for a burean of our Government to supplement the work of the German duo, by adding the all-important factor of quantity. The complete exposition of the exact amounts of the many synthetic dyes required to meet the almost numberless needs of a population of over 100,000,000, portrays approximately the relative demands of all other nations with highly organized textile and allied interests. The young American dyestuff industry, now in a position to expand rapidly and to embrace in its scope the great majority of the colors in current use, will naturally find in it a sure guide for coördinating the diverse phases of manufacture, establishing the capacity of units, and shaping all plans for harmonious expansion.

More than this, it will be of almost equal value to those seeking to create the national coal-tar industries of Great Britain, France, Russia and Italy. Even the newly organized industry in Japan may profit from its summaries, although in a less pronounced degree, on account of the widely divergent taste for colors between the Orient and the Occident.

Should China plan to manufacture her own coal-tar dyes, but little help could be secured from this compilation in formulating schemes for installing plants. Synthetic indigo constitutes two-thirds of the Chinese consumption of artificial colors. It enters to the extent of 14 per cent into the Japanese imports of dyestuffs, and forms but ro per cent of the American consumption.

One of the first results of the compilation of this census was to show how exceedingly vague an idea of the extent to which synthetic dyes are consumed in the United. States prevailed in commercial and manufacturing circles. Those most closely in touch with the branch have estimated hitherto that the annual American consumption of coal-tar colors did not exceed 20,000 tons. As a matter of fact, it is nearly one-half again this amountmore exactly, 29,000 short tons.

SUMMARY OF THE MOST IMPORTANT COLORS IMPORTED

It has seemed desirable, in the interest of American chemists who are now studying closely the problems connected with the creation of a national dyestuff industry, to prepare a summary of the more important synthetic colors currently imported into this country and compiled systematically, in the complete census of dyes consumed in the United States, now in press.

The list prepared (pages 1042,3 and 4 ) includes practically all colors, the annual importation of which, during the fiscal year I $913-14$, exceeded in amount 10,000 lbs. These colors, for the sake of classification, are divided into six groups, according to the amount of the importation.

PRICES OF DYES

The values of the different colors imported from Europe are taken directly from the invoice entries. The prices varied but little during the course of the fiscal year $\mathrm{rg}^{1} 3^{-14}$. They are on file in the Bureau of Foreign and Domestic Commerce. It did not seem necessary to reproduce them in full in the enumeration of colors. The average price for the year, in the case of any dyc, is easily ascertained by a simple act of division.

In most cases the values stated represent the lowest possible estimate of wholesale cost which can be placed upon the wares in question. The bulk of the importations is shipped from the great 
manufacturing firms of Germany, Switzerland and England, to their agents in this country. The latter are ordinarily incorporated American companies, bearing essentially the same names as the European houses which they represent, and may be practically under the control of the latter, if not financed by them directly.

Under these circumstances and in view of the exceptional diffculty of ascertaining market values for the highly differentiated gradations of quality in the thousands of brands of artificial colors, there was a strong temptation to place the lowest possible values upon wares subject to an ad valorem duty of 30 per cent.

It is doubtful in many cases, therefore, whether the values published in the following lists are fully equal to those against which American manufacturers of colors would contend, should all the factors, falling under the head of "unfair competition," be eliminated in the international trade in synthetic dyes. In these values there is a slight element of variability and uncertainty, based upon the lack of uniformity in invoicing colors.

In some cases-probably the majority-the prices and values are net, not covering charges for containers and packing, freight, and insurance to seaport, consular certification, minor shipping charges at point of departure and at seaport. Wares shipped by the "Badische Co.," the Berlin "Actien-Gesellschaft," the "Cassella Co.," the "Griesheim-Elektron," and the "Chemikalienwerk-Griesheim" are usually invoiced in this manner.

Other firms, such as the "Bayer Co.," the "Sandoz Co.," "Carl Jäger," and "Beyer and Kegel," nclude in their prices the cost of containers and packing, freight and insurance to seaport, consular certification, and minor shipping charges. In other words, their prices are f. o. b. ocean steamers at Hamburg, Bremen, Rotterdam, Antwerp, ete.

It has not been possible, on account of time limitations, to estimate this small factor in the case of each shipment and make the accompanying correction in value so as to have actual uniformity in the basis of valuation. The element of quantity is the dominant feature in this work, and in view of what is stated above, very elaborate calculations of values would be of doubtful utility. COST OF PACKING, FREIGHT, INSURANCE, ETC.

It is well, however, to know with some approximation to exactness the extent and nature of the charges incident to the importation of European coal-tar colors into the United States. They are as follows:

Consular certification-A fee of $\$ 2.50$ for all invoices covering shipments, the value of which exceeds $\$$ IOO.

Freight to seaport-This is quite variable, depending upon the distance to be traversed and whether rail or water transportation is employed. Rates per $\mathrm{lb}$, (net) of color are \$0.00125 from Berlin; \$0.0056 to \$0.007 I from Basel; \$0.0008 to \$0.002I6 for points on the Rhine.

Insurance, in Iransit :o seaport--Rates vary from $\$ 0.0007$ to \$o.00I6 for each dollar of invoice value.

Shipping charges-The item appears occasionally and ranges from $\$$ I to $\$ 2$ for each $\$ I, 000$ of an invoice.

Ocean freight--Customary rates from European ports, such as Hamburg and Antwerp to New York, were, prior to the war, $\$ 9.75$ per metric ton (gross weight) for most of the coal-tar dyes, and \$7.9I for certain categories, such as sulfur black and other sulfur colors.

Several large invoices of colors showed an average rate for ocean freight per net $1 \mathrm{~b}$. of dyestuff, of $\$ 0.0047 \mathrm{I}$, ranging from $\$ 0.004125$ to $\$ 0.00539$. Through freight from Frankfort to New York on a large and varied assortment of artificial dyes was at the rate of $\$ 0.00755$ per net $\mathrm{lb}$. of colors.

On an average, I $1 \mathrm{~b}$. of color (net) is represented by $1.165 \mathrm{lbs}$. gross weight, the tare per $\mathrm{lb}$. ranging from 0.155 to o.I $74 \mathrm{~b}$. on shipments of some size

Marine insurance-Insurance on large shipments of dyes from Frankfort to New York, covering both inland and marine insurance, was equivalent to $\$ 0.000916$ per $1 \mathrm{~b}$. (net) of colors.
This represented $\$ 0.00497$ on each dollar of value, or about 0.5 per cent on the value. Shipments by another company, located on the Rhine, averaged 0.3 per cent.

Packing-There is some diversity in the average cost of containers. This item ranges from $\$ 0.00459$ to $\$ 0.00863$ per $1 \mathrm{~b}$. (net) of color in a number of large invoices. A fair average would be $\$ 0.0065$ I per net $1 \mathrm{~b}$.

Some of the more expensive colors are shipped in tin boxes, packed in cases holding Ioo lbs. (net). Cases cost $\$ 0.48$. The charges for tins are as follows: I 1b., $4.8 \mathrm{c}$.; 5 lbs., 9.6c.; ro lbs., I5.5c.; 25 lbs., 32c. Kegs, containing roo lbs., cost usually $\$ 0.95$, but range in value from $\$ 0.63$ to $\$$ I.I4. Casks hold ordinarily about $500 \mathrm{lbs}$. The net contents range, however, from $4 \mathrm{I} 5$ to $595 \mathrm{lbs}$. In four large shipments the average net weights were $442,469,480$, and $53 \mathrm{I}$ lbs. The general average was 480 lbs. The average price of casks is $\$ 1.90$. They range, however, in cost from $\$$ I.55 to $\$ 3.24$.

On an average the importation of European colors to New York costs $\$ 0.014$ per net $\mathrm{lb}$. for packing and transportation (packing $\$ 0.0065$, transportation $\$ 0.0075$ ), and $\$ 7$ per $\$ 1,000$ of value for insurance and incidental charges (insurance $\$ 5$, shipping charges and consular certification $\$ 2$ ).

American consumers of coal-tar colors, who may wish to compare the prices paid by them two or three years ago for European wares with the prices based upon the values furnished in this report, can add to the prices calculated from these values the cost of the above items. In addition there comes the duty of 30 per cent ad valorem on all artificial colors, except indigo and its derivatives, and colors made from anthracene (largely alizarin) and carbazole, which were exempt from duty under the late tariff. This duty is levied upon the combined cost of a dye and its containers. Furthermore, the normal cost of handling, storing, and distributing, in the importing houses, is to be added. The difference between the sum total of these various items and the current price for a given color represents the profit made by the importer.

ARTIFICIAL COLORS MANUFACTURED IN THE UNITED STATES

The manufacture of coal-tar colors in the United States has been in existence for some 37 years. Prior to I9r 5 it had never become a factor of importance in supplying the American market. The reasons for this slowness of development have been presented in detail in the monograph published in I 9 I 5 by the Bureau of Foreign and Domestic Commerce, entitled "Dyestuffs for American Textile and Other Industries" (Special Agents Series No. 96).

The American manufacture was confined almost entirely to the "assembling" into finished dyes of coal-tar intermediates imported from Europe, chiefly from Germany. In its entirety it represented less than one-tenth of the activity to be encountered in any one of the larger companies producing synthetic colors in Germany and Switzerland.

\section{STATUS OF THE DOMESTIC PRODUCTION IN I9I4}

The status of the industry for the calendar year 1914 is shown by the following tabular statement prepared by the Bureau of the Census.

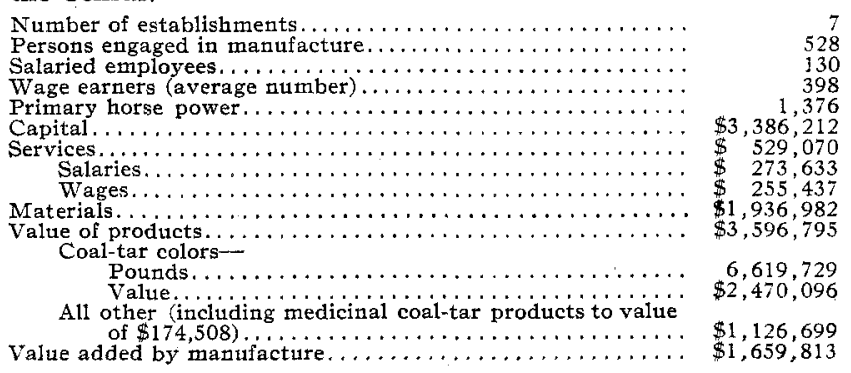

The scope and extent of the manufacture carried on prior to the war by the seven American companies engaged in this branch 
are summarized under their respective names. No attempt has been made to estimate the annual output of the individual colors made in the American factories, as it fluctuated largely from year to year.

In nearly all cases the character of the manufacture has been vastly affected by the conditions prevailing since I9I4. As a rule the variety of colors has been diminished while the output has been vastly algmented.

The number of employees has been notably augmented. In general, it has been quintupled. In one case the force is 50 times greater than in 1914 .

THE SCHOELIKOPF ANILINE AND CHEMICAI, WORKS (INC.)

This firm, located at Buffalo, N. Y, was founded in 1879 , and is the oldest American company in this industry. A number of dyestuffs in current use originated in its laboratories. It has shown a commendable degree of enterprise in maintaining its position for over a third of a century, frequently under conditions of a most discouraging nature. It has also earned the grateful recognition of a multitude of American consumers of dyes by swiftly enlarging the capacity of its works so as to alleviate materially the severity of the dyestuff famine to which our textile and allied interests have been exposed.

The annual output of this firm constituted about one-half of the American production of coal-tar dyes. The following colors were currently mantufactured before the war. The serial numbers correspond to those given in Schultz's "Farbstofftabellen:"

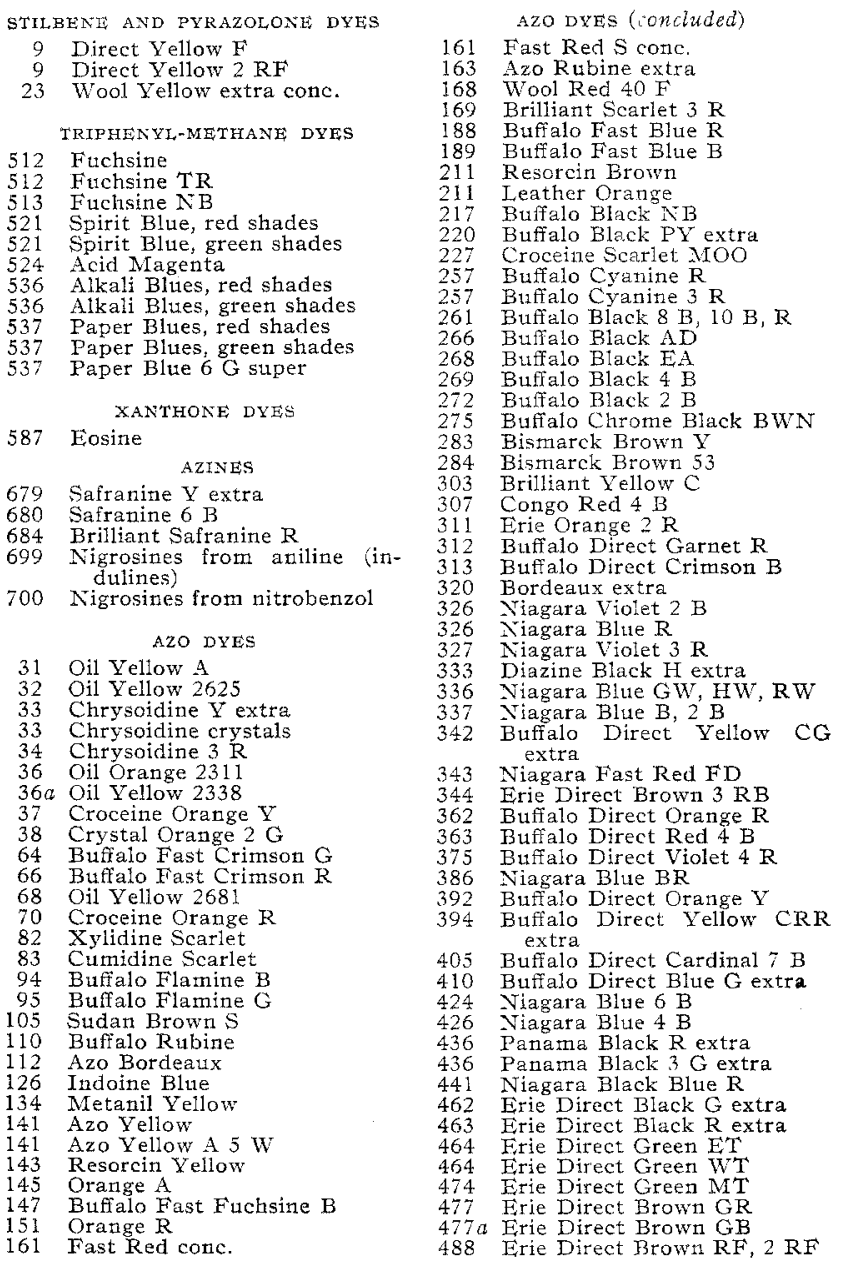

THE HELLER AND MERZ CO.

This firm, located at Newark, N. J., stands second in point of seniority and importance. Its annual output of coal-tar colors was estimated at slightly less than one-quarter of the country's production. It has catered very largely to the needs of the paper trade. In addition to organic dyestuffs, it has manufactured large quantities of mineral colors, notably ultramarine. The equipment for the production of coal-tar dyes has been largely augmented during the past year.

The following artificial colors were currently manufactured in 1914 (serial numbers of Schultz's Farbstofftabellen):

$$
\begin{aligned}
& \text { AZO DYES } \\
& 33 \text { Chrysoidine } \\
& 145 \text { Orange II } \\
& 698 \text { Nigrosine } \\
& \text { spirit-solub } \\
& \text { Nigrosine, } \\
& \text { water-soluble }
\end{aligned}
$$

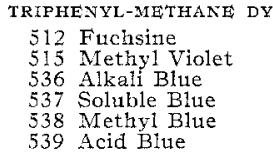

THE BAYER CO. (INC.)
This company owns works at Rensselaer, N. Y., where several of the staple colors and medicinal products of the Farbenfabriken vormals Friedr. Bayer \& Co., of Leverkusen, Germany, are manu. factured on a scale of some importance. The output of coaltar dyes prior to the war constituted somewhat less than onefifth of the entire production. The expansion during the past year and a half has not been as pronounced as in the case of the other establishments.

Prior to the war the company manufactured the following colors:

AZO DYES

33 Chrysoidine
283 Bismarck Brown

TRIPHENYL-METHANE DYES
512 Fuchsine
536 Alkali Blue
537 Soluble Blue

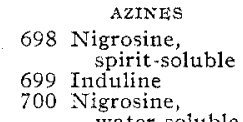
699 Induline water-solub

W. BECKER'S ANILINE AND CHEMICAL WORKS (INC.)
s company, founded in I $9 \mathrm{I} 2$, is located at Brooklyn, $\mathrm{N} . \mathrm{Y}$. Prior to the war it specialized on alizarin substitute colors. The annual output was modest, estimated at about 180 tons. During the past I 8 months the plant has been rapidly enlarged. To-day it is second in importance as a factor in the domestic color industry.

The following colors were manufactured regularly prior to I9I5:

AzO DY Alizarin Yellow FF
83 Ponceau 3 R
145 Orange II
163 Azo Rubine WB
166 Fast Red A
188 Acid Fast Blue
SR

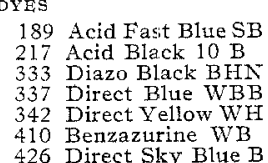

XANTHONE DYES 599 Chrome Blue R, powder an d paste

626 ChATINE DYES B B paste Blue $B$

THE CENTRAL DYESTUFF CO.

This company, located at Newark, N. J., was founded in 1898 . The output was not large, possibly 4 per cent of the country's production. It included, however, several dyes of importance for the textile industries. The plant has been notably enlarged during the past year.

Prior to the war the following colors were currently manufactured:

$\begin{array}{llr} & & \\ 31 \text { Amido-azo- } & \text { AzO DYES } & 697 \text { Enduline } \\ \text { benzene } & 161 \text { Fast Red } & 698 \text { Nigrosine } \\ 33 \text { Chrysoidine } & 163 \text { Azorubine } & \\ 37 \text { Crosceine Orange } & 168 \text { Amaranth } & \\ 68 \text { Amido-azo-toluene } & 223 \text { Scarlet } & \\ 112 \text { Bordeaux B } & 232 \text { Sudan III } & \\ 145 \text { Orange II } & 283 \text { Bismarck Brown } & \end{array}$

THE CONSOLIDATED COLOR AND CHEMICAL CO.

This company, located also at Newark, N. J., manufactured for some years prior to the war less than roo tons annually of colors. The following colors for textile works were currently produced.

58 Alizarin Yellow R. 168 Fast Red 144 Naphthol Orange

In addition, a certain variety of colors for pigments, especially alizarin, para and scarlet lakes, and for use in paper making, were regularly manufactured. During the past year the plant has been greatly enlarged. It is at present an important center of production.

HUB DYESTUEF AND CHEMICAL CO

This company, located at South Boston, Mass., manuiactured on a modest scale, for a few years, prior to I9r 5,58 Alizarin Yellow R.

IMPORTATION OF COAL-TAR CRUDES AND INTERMEDIATES

As stated above, the synthetic colors manufactured in the United States prior to I 9 I 5 were made almost exclusively from intermediate coal-tar products imported from Europe. Germany was the chief source. A considerable amount, however, was of British origin.

A single noteworthy exception is found in the case of aniline. The American manufacture of this all-important intermediate was organized in 1910 . The annual output had attained about 900 short tons in 1914 -the product of a single establishment. 
At present aniline is regularly manufactured by over 30 companies and the annual output is in excess of 16,000 short tons.

In connection with the preceding enumeration of the artificial dyes currently produced in the United States prior to the war, it is of considerable importance to know what coal-tar crudes and intermediates were imported for use in their manufacture and, further, the quantity and value of each crude and intermediate. This information is furnished in the following tabular statement based upon the importations for the fiscal year ending June 30 , I9I4:

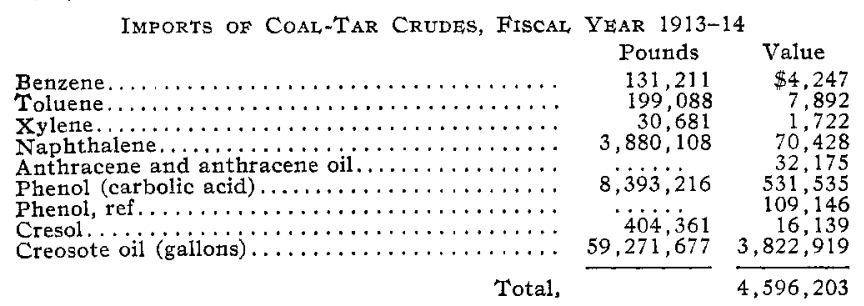

An exceedingly small part of the above-mentioned coal tar crudes was utilized in the manufacture of artificial colors. The creosote oil was employed in the preservation of timber. The phenol served chiefly as a disinfectant and antiseptic. The chief use of naphthalene was in the familiar form of "moth balls."

During the calendar year r913, according to official German records, the following coal-tar crudes were imported into the United States from Germany: Naphthalene, 7,900 short tons; carbolic acid (pure and crude), I,320 short tons; cresol, 220 short tons; creosote oil, 17,600 short tons.

\begin{tabular}{|c|c|c|}
\hline $\begin{array}{l}\text { INTERMEDIATES } \\
\text { NITRO COMPOUNDS }\end{array}$ & Pounds & Value \\
\hline 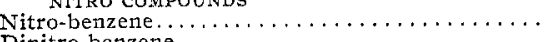 & $1,087,911$ & $\$ 59,835$ \\
\hline 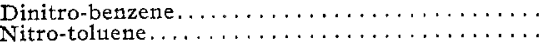 & 164,650 & 10,399 \\
\hline 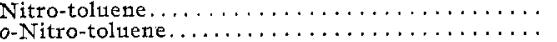 & $\begin{array}{r}6 \\
42\end{array}$ & $\begin{array}{r}359 \\
2.969\end{array}$ \\
\hline$\ldots \ldots \ldots \ldots$ & 547,701 & $\begin{array}{r}2,969 \\
36,660\end{array}$ \\
\hline$\ldots \ldots \ldots \ldots \ldots \ldots \ldots \ldots \ldots$, & 57,242 & 13,24 \\
\hline 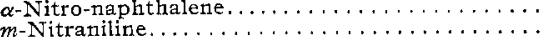 & & \\
\hline ( & 506,961 & $\begin{array}{r}1,037 \\
67,638\end{array}$ \\
\hline$\ldots \ldots \ldots \ldots \ldots \ldots \cdots \cdots$ & & \\
\hline $\begin{array}{l}\ldots \ldots \ldots \ldots \ldots \ldots \ldots \ldots \ldots \\
\cdots \ldots \ldots \ldots \ldots \ldots \ldots\end{array}$ & & \\
\hline (n... & 20,7 & $\begin{array}{l}4,200 \\
6,524\end{array}$ \\
\hline 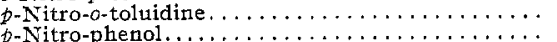 & 30, & 9,723 \\
\hline$\cdots \ldots \ldots \ldots$ & & \\
\hline ICIDS & & \\
\hline$\ldots \ldots \ldots \ldots \ldots \ldots \ldots$ & & $\begin{array}{l}51,701 \\
15,597\end{array}$ \\
\hline 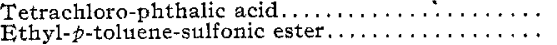 & & 659 \\
\hline man & & \\
\hline$\ldots \ldots$ & 1,4 & \\
\hline (n) & $3,083,4$ & $\begin{array}{l}116 \\
222\end{array}$ \\
\hline , n & 1,0 & \\
\hline$\ldots \ldots \ldots$ & 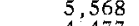 & 365 \\
\hline$\ldots \ldots$ & $\begin{array}{r}4,477 \\
108,835\end{array}$ & 257 \\
\hline 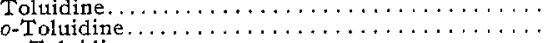 & 309,595 & $\begin{array}{l}14,161 \\
27,361\end{array}$ \\
\hline$\ldots \ldots \ldots \ldots \ldots \ldots \ldots$ & & \\
\hline$\ldots \ldots \ldots \ldots \ldots \ldots \ldots \ldots \ldots$ & & 4,104 \\
\hline , n & 18 & 2,167 \\
\hline$\cdots \cdots$ & 25 ; & $\begin{array}{r}11,925 \\
2,705\end{array}$ \\
\hline 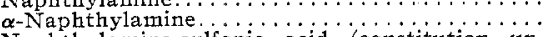 & 112 & 10,620 \\
\hline -sulfonic acid (constitution un- & & \\
\hline ne stilfonic acid (I acid; 1,5$) \ldots$ & 2,832 & \\
\hline$\ldots \ldots \ldots \ldots \ldots$ & & \\
\hline disulfonic acid (Freund; $1,3,6$ ) & 5,2 & $\begin{array}{l}71 \\
60\end{array}$ \\
\hline$\alpha$-sulfonic acid ( $\alpha$ or Badische & & \\
\hline & 23,2 & 7,579 \\
\hline & 2,316 & 495 \\
\hline $\begin{array}{l}\text { aphthylamine-disulfonic acid }(\alpha \text { or } R \text { acid; } \\
\text { amido-R-salt; } 2,3,6)\end{array}$ & 46,267 & 4,495 \\
\hline $\begin{array}{l}\text { aphthylamine-disulfonic acid }(\gamma \text { or } G \text { acid; } \\
\text { amido- } G \text {-salt } ; 2,6,8) \ldots \ldots \ldots \ldots \ldots \ldots \ldots\end{array}$ & 3,603 & 230 \\
\hline INES AND DERTVATIVES & 48, & \\
\hline & 55,5 & 9, \\
\hline - $-2=-$ & 1,102 & \\
\hline 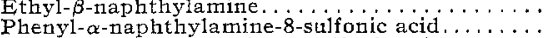 & 9,1 & \\
\hline & & \\
\hline
\end{tabular}

DIAMINES AND DERIVATIVES

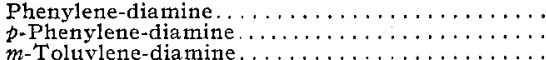

m-Toluylene-diamine...............
m-Toluylene-diamine-sulfonic acid $(1,2,4,6) \ldots \ldots$

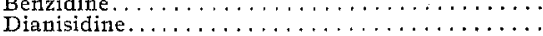

PHENOLS AND DERIVATIVES

Salicylic acic

Acetyl-salicylic acid

Resorcin ...........

Pyrogallol........

Gallic acid $\ldots \ldots \ldots$
Naphthols $(\alpha$ and $\boldsymbol{\beta})$
$\alpha$ Naphthol........

$\alpha$ Naphthol ............ phthol-5-sulfonic acid ( $L$ or Cleve's acid -Naphthol- $3,6,8$-trisulfonic acid $(1,3,6,8) \ldots \ldots$

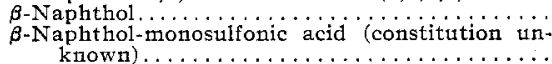

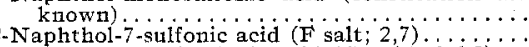
$\beta$-Naphthol-7-sulfonic acid (F salt; 2,7$), 3,6, \ldots$
$\beta$ Naphthol- 6,8 -disulfonic acid (G acid; $2,6,8)$

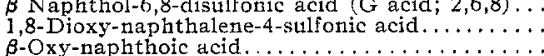

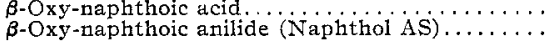
AMIDO-PEEENOLS AND DERTVATIVES

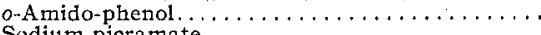
Oxy-nitraniline......

Diamido-phenol

p-Amido-phenol hydrochioride

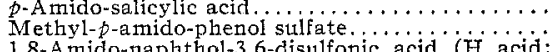

1,8-Amido-naphthol-3,6-disulfonic acid (H acid;

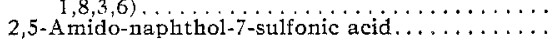
ALDEHYDES AND QUTNONES

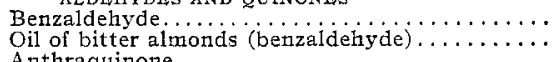

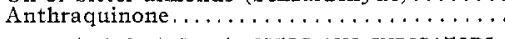

DEVELOPERS, REDUCERS AND INDICATORS

Fast Bhue Developer AD (amido-diphenylamine)

Oxamine Developer

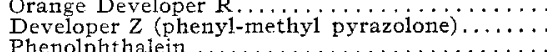

Total .......................

37,907

11,088

2,355
55,245
5,256

10,656

$\$ 7,704$

25,582

16,991

18,82

61,624

66,596

61,635
70,469

70,469
44,089

25,126

6,443

$1,030,268$

32,852
1,996

11,996

2,178
2,359
2,997

4,425
11,873

18,175
25,140

25,140

20,429

4,193
2,271

5,026

1,344
74,238

5,998

1,404

1,056

1,218

625
5,207
200

223
485
32
391

$\begin{array}{rr}200 & 341 \\ 10,631 & 1,684 \\ 652 & 189\end{array}$

9,188

10,582

2,996
13,658

96,296
1,153

22,168

12,950

7,525
25,193

2,757

\begin{tabular}{rr}
100 & 39 \\
11,096 & 2,119 \\
1,301 & 376 \\
14,397 & 377 \\
\hline & 14,090 \\
\hline
\end{tabular}

$\overline{10,165,896} \overline{\$ 1,082,775}$

\section{SUMMARY OF IMPORTS OF COAL-TAR PRODUCTS, I9I3-I4}

The coal-tar crudes and intermediates listed above, with the exception of aniline oil and salts, several acids, such as carbolic acid and salicylic acid, alizarin and colors derived from alizarin, anthracene and carbazole, indigo and its derivatives, and a few other compounds, such as antipyrine, aspirin, saccharin, and phenolphthalein, are included in the following summarized statement of the imports for consumption into the United States of coal-tar products, for the fiscal year ending June 30 , I9I4, published by the Bureau of Foreign and Domestic Commerce.

Imports designated by an asterisk (*) are for the period July 1 to of the fiscal year.

\section{Coal-'Tar Products}

Anthracene and anthracene oil.

Colors or dyes, n. s. p. f.

Do. (for tse of the United States).

Dead or creosote oil ( $59,271,677$ gallons)

All other, not medicinal and not colors or

dyes, known as benzol, toluol, thaph-

thalin, xylol, phenol, cresol, toluidine,

xylidin, cumidin, binitrotoluol binitro

benzol, benzidin, tolidin, dianisidin,

naphthol, naphthylatnine, diphenyl

amin, benzaldehyce, benzylchloride,

resorcin, nitrobenzo], and nitrotoluol

foacids and amidonaphtholsulfoacids

and their sodium or potassium salts

amidosalicylic acid, binitrochlorbenzol

diamidostilbendisulfoacid, metanilic
acid, paranitranilin, and dimethylacid, paranitranilin, and dimethyl-
anilin.

Distillates, n. s. p. f., not medicinal and not colors or dyes, benzol, naphthol resorcin, toluol, xylol.
Not medicinal and not colors or dyes, known as toluidine, xylidin, climidin, binitrotoluol, binitrobenzol, benzidin, phenylamin, benzaldehyde, benezyl chloride, nitro-benzol, and nitrotoluol naphthylaminsulfoacids, naphtholsulfoacids, and amidonaphtholsulfoacids and their sodium or potassium salts armidosalicylic acid, binitrochlorbenzol diamidostilbendisulfoacid, metanilic acid, paranitranilin, and dimethyl-
anilin.

\begin{tabular}{lc} 
Rate of duty & \multicolumn{1}{c}{ Value } \\
Freet & $\$ 32,175.00$ \\
30 per cent & $7,537,869.55$ \\
Free & $3,822,919.00$ \\
Free &
\end{tabular}

Free*

$288,799.00$

5 per cent $\uparrow$

$138,636.00$ 


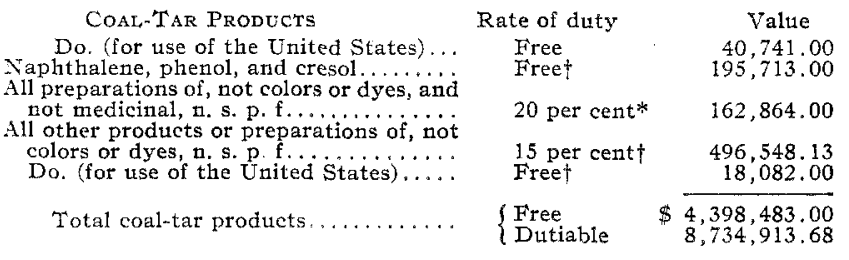

IMPORTS OF INTERMEDIATES FROM GERMANY

The official records of exports of intermediates from the German Empire to the United States show that the following quantities, in short tons, were shipped during the calendar year I9I3:

Aniline oil and salts.........................

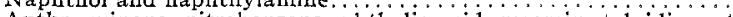

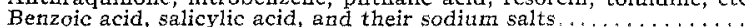

Total (short tons $\}, \ldots \ldots \ldots \ldots \ldots \ldots \ldots \ldots, \overline{4,664}$

THE MARKS OF COAL-TAR COLORS

The great diversity of marks employed by the different manufacturers of artificial dyes is a source of confusion and bewilderment to many, especially to those in the United States now taking an active interest in the evolution of a domestic coal-tar chemical industry, and but slightly familiar with the commercial features of this most complicated of all the varied branches of technology. As the subject has never been treated to any extent in the literature devoted to this industry, it seems desirable to furnish some information of a general character, as explanatory of the symbols used to discriminate between the different shades of dyestuffs.

Several of the managers and chemists of the leading firms in New York City devoted to the sale in this country of the dyes, tabulated in the census, have kindly responded to inquiries in this connection. The following excerpts from their letters furnish a fairly good portrayal of the few conventional features, and the general lack of uniformity characterizing the use of marks for coal-tar colors

The customary designation of dyestuffs as practiced by the manufacturers, their representatives, and the free-lances in the dyestuff business does not appear to be governed by any set formula. In the early days of the artificial-dyestuff industry, when dyes were few, the manufacturers designated distinct differences in shade by the letters $\mathrm{B}, \mathrm{R}$, and $\mathrm{G}$, signifying blue, red, and yellow (gelb). Our French confrères used the corresponding $B, R, J$ (jautue, and $V$ (vert), which became familiar to dyers
and dyestuff users. As dyes multiplied, differences in shade beca me more and dyestuff users. As dyes multiplied, differences in shade became more
numerous and it became necessary to alter or to augment, as the case numerous and it became necessary to alter or to augment, as the case
might be, the distinguishing marks. Consequently the marks $2 \mathrm{~B}$, or $2 \mathrm{R}$, might be, the distinguishing marks. Consequently the
or $2 G$, etc. became common and continue to this day.

$2 \mathrm{G}$, etc., became common and continue to this day.
No uniformity, however, exists between the corresponding marks of different concerns. For example, Chicago Blue $6 \mathrm{~B}$, the lightest and firm as $7 \mathrm{~B}$, while among the products of still another firm it is indicated as FF. different houses. One skilled in the art of buying and selling dyestuff can, without great difficulty, distinguish a competitor's types among a variety of designations.

As far back as 1888 I went into this matter of type designations, discussed it with prominent dye agents, and was supplied by them at the time with comprehensive lists of their dyes and the meaning of their distinguishing letters. These demonstrated conclusively that each firm selling dyes was a law unto

its products was concerned.
Many individuals in and out of the textile industry and dabblers in Many individuals in and out of the textile industry and dabblers in
tinctorial chemistry have entertained the idea that there was a key to a tinctorial chemistry have entertained the idea that there was a key to a
prescribed code of type designations which, in the hands of one possessing prescribed code of type designations which, in the hands of one possessing
it, would unlock the nuumerous combinations of dyes required by individual customers, but this is not so.

We have, for example, a familiar instance of one of the smaller dyestuff firms, which designates its dye mixtures by numbers preceded by letters. These letters indicate the mixture books, Volume A, Volume B, Volume C, etc., and the number is that of the mixture. In order to duplicate the mixture, as in the case of a physician's prescription, one simply goes to the volume indicated by the letter and picks out the corresponding number. For all time that number holds good for one particular customer. No

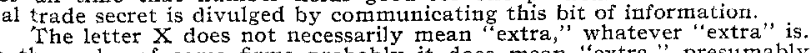
It the codes of some firms probably it does mean "extra," presurnably higher strength or greater brilliancy or solubility. In other cases it may casignate the region where certain dyes are sold. N , for example, ndiYork).

The whole subject of dye designations is so complex that it becomes a hopeless problem to untangle. General references to it in literature are scarce, for the reason that there is nothing definite to say upon the subject. It is much like patent medicines; the
code to designate such remedial a aents.

code to designate such remedial agents. Some dyestuffs are differentiated irom others by appended letters to
indicate their source, or the materials of which they are prepared. For instance, one of the well-known commercial types of Methylene Blue-
specifically Methylene Blue SZ--of no particular merit as to shade, possesses properties peculiar to itself on account of having been prepared free from zine (sans zinc)

desination the foring it is easily realized that the matter of commercial designations of dyes is very complex. While there are standards, $i$. e., established types of individual dyes, such as those enumerated in the Schultz tables, from which millions of combinations are possible, every
dyestuff firm has thousands and thousands of these combinations upont their books. Each is necessarily designated by some intelligible and combprehensive system in order to guard against errors and mistakes in compotunding when called for.

Many wel1-known types of "straight" dyes as produced by the manufacturer are, per se, of little value when used alone. Their value is brought out when used in combination with other dyes, and this is the strong point of many valuable mixtures that under no circumstance can be replaced by "straight" dyes. While we prefer to une straight or unmixed colors, we are frequently compelled to make use of mixtures, the value of which for dyeing purposes far outweighs the usefulness of the individual components when used separately. Such mixtures, of necessity, tnust be designated by
different letters or numbers, to prevent confusion. A common instance different letters or numbers, to prevent confusion. A commonon instance
of this is the very extensive series of combination shades, of great value, of this is the very extensive series of combination shades, of great value,
produced with the fast reds and azo scarlets, which may or may not be produced with the fast reds and azo scarlets, which may or may not
modified in tone by the judicious admixture of acid violets.- $L . J . M$.

There is no uniform practice and not much of a system in the marks which are used to distinguish the different brands of dyestuffs. This fact may seem rather strange, but it may be readily understood if one realizes that all dyestuff concerns have manufacturing and selling ends
of the business- two loosely connected departments. The dyestuff is sent from the manufacturing department to the dyehouse, which is connected with the selling department. Both the dyehouse and the selling department are kept in ignorance regarding the clemical nature of any
new product which is brought out. The same has, therefore, to be classified new product which is brought out. The same has, therefore, to be classified according to its shade and its dyeing. properties. The result is that in many cases the dyer identifies a color with a group of others having simnilar The leading principle in naming the colors is less one of general classification
than to furnish the salesman of a special factory with some hint as to the

dyeing properties of any given color.

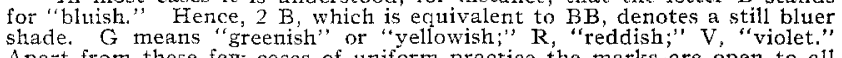
A part from these few cases of uniform practice the marks are open to all
kinds of explanation, "The matk $L$, for instance, may mean "soluble," or kinds of explanation. The mark L, for instance, may mean "soluble," or "ast to light, or even may stand for Lud wigshaven, which means that the
color in question is identical with a well-known product of the Badische Co. at Ludwigshavent. In the same way C may stand for "Cassella" Meister, Lucius \& Brüning, Hoechst a. M.); F for "Elberfeld" (Farbenfabriken vorm. Friedr. Bayet \& Co., Leverkusen, formerly at Elberfeld); fand B or A for "Berlin" (Actien-Gesellschaft fur Ant Anlin-Fabritiation,
anerlin) All refer to types against which the competitive products have been stan

The word "extra" indicates either a special shade or a special concentra tion. It is a rule with 11 that our "extra" marks are more concentrated than the single brands, which otherwise bear the same name and mark. The letter $X$ is used by other firms in the same way as our "extra," for
higher concentrations, without, however, giving any definite information regarding the proportionate strength.

Many suggestions have been made in order to do away with this rather confusing habit of classification, but so far without success. The reason is that the selling staff on the one hand, and the millman on the other hand, are not expected to possess much chemical or coloristic knowledge. Such
knowledge only would enable them to benefit from a more complicated and scientific systern, - $A$. $M$.

It is a pretty well-established practice among dye manufacturers to use certain marks and letters in connection with the name of any color to yellow, and this sariation from the standard type is designated by the letters $B, G, R$, etc.

Tale, for instance, Methy1 Violet, which varies in shade from $3 \mathrm{R}$ to $6 \mathrm{~B}$, The $3 \mathrm{R}$ indicates a reddish shade nearly approaching Magenta, and $6 \mathrm{~B}$ indicates a bluish shade nearly approaching a product like Victoria Blue $B$. It follows from this that $3 \mathrm{R}$ means a tint redder than $2 \mathrm{R}, 3 \mathrm{~B}$ " means a tint bluer than $2 \mathrm{~B}$, and $6 \mathrm{~B}$ denotes a still bluer shade thann 3 B. "Gelb," which means yellow. The French word for yellow is "jaune." Consequently, French, Belgian, and sometimes Swiss firms use the lette $\mathrm{J}$ where Germans use $\mathrm{G}$. English and Americans employ for the same purpose the letter $\mathrm{X}, 2 \mathrm{G}$ means the same thing as if the letter $\mathrm{G}$ is re-

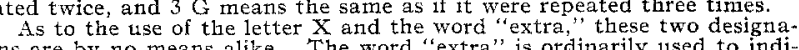
tions are by no means alike. The word "extra" is ordinarily used to indicate a quality superior to the regular type. This is sometimes shortened simply to the letter $X$. More generally $X$ indicates that the phoduct in question is reduced 10 per cent below the standard type. XX in that case would mean that it is reduced 20 per cent below the standard type. " wool," and $\mathrm{TW}$ mark $\mathrm{W}$ indicates that a dye is emp

and FW refers to "half wool" or union fabrics. Alizarin Blue $S$, SR, and SW. Sometitues it denotes a sulfonic acid, as WT. $-E . C . K$. $K$.

WASHINGTON, D. C.

\section{CONDITIONS AFFECTING THE ESTABLISHMENT OF THE NITRATE INDUSTRY IN THE UNITED STATES ${ }^{\prime}$}

BY IAWRENCE ADDICKS

The general problem of the establishment of the nitrate in dustry in the United States is complicated by the existence of several more or less conflicting and unrelated factors which fact in large measure accounts for the present confusion as to the relative merits of the various available processes for the production of nitric acid. These factors may be stated as difficulties in

1 Read before the 53rd Meeting of the American Chemical Society, September 25-30, 1916 